\title{
Genome-wide analysis of the endoplasmic reticulum stress response during lignocellulase production in Neurospora crassa
}

\author{
Feiyu Fan ${ }^{1,2}$, Guoli Ma ${ }^{1,2}$, Jingen Li ${ }^{1,2}$, Qian Liu', Johan Philipp Benz ${ }^{3}$, Chaoguang Tian ${ }^{1 *}$ and Yanhe Ma ${ }^{1}$
}

\begin{abstract}
Background: Lignocellulolytic fungal cells suffer endoplasmic reticulum (ER) stress during lignocellulase synthesis; however, an understanding of this integrated process on a genome-wide scale remains poor. Here, we undertook a systematic investigation of this process in Neurospora crassa ( $N$. crassa) using transcriptomic analysis coupled with genetic screens.

Results: A set of 766 genes was identified as the ER stress response targets (ESRTs) in N. crassa under cellulose utilization conditions. Among these, the expression of 223 and 186 genes showed dependence on IRE-1 and HAC-1, respectively. A total of 527 available mutants for ESRT genes were screened, 249 of which exhibited ER stress susceptibility, including 100 genes with unknown function. Disruption of ire-1 or hac-1 in N. crassa did not affect transcriptional induction of lignocellulase genes by cellulose but severely affected secretion of the corresponding enzymes. A global investigation of transcription factors (TFs) discovered three novel regulators (RES-1, RES-2, RRG-2) involved in lignocellulase secretion. Production of lignocellulases in $\Delta$ res- 1 increased by more than $30 \%$ in comparison to wild type (WT), while secretion decreased by nearly 30\% in strains $\Delta$ res-2 and $\Delta$ rrg-2. Transcriptional profiling of the three TF mutants suggests they are deeply involved in lignocellulase secretion and ER stress response.

Conclusions: Here, we determined the transcriptional scope of the ER stress response during lignocellulase synthesis in the model cellulolytic fungus $N$. crassa. Through genome-wide mutant screening and analysis, dozens of novel genes were discovered to be involved in the process. The findings of this work will be useful for strain improvement to facilitate lignocellulase and biomass-based chemical production.
\end{abstract}

Keywords: ER stress, UPR, Transcription factor, Lignocellulase secretion, RNA-seq, RESS

\section{Background}

Saprophytic fungi evolved a highly efficient capability to secrete enzymes into their extracellular matrix to synergistically depolymerize biomass. This natural property has been exploited for lignocellulase production in industry [1]. Although Trichoderma reesei (Hypocrea jecorina) and Aspergillus spp. have been successfully developed as systems for lignocellulase production, a clear genetic basis underpinning the requirements of effective secretion has not yet been elucidated [2]. Early electron microscopy analysis revealed that endoplasmic reticulum (ER) proliferation

\footnotetext{
* Correspondence: tian_cg@tib.cas.cn

'Key Laboratory of Systems Microbial Biotechnology, Tianjin Institute of Industrial Biotechnology, Chinese Academy of Sciences, Xiqi Dao32, Tianjin Airport Economic Area, Tianjin 300308, China

Full list of author information is available at the end of the article
}

occurred in the T. reesei hyper-secretion mutant RUT-C30 compared with its parental strain, QM6a [3,4]. Further characterization of the RUT-C30 strain revealed the transcript levels of ER-resident chaperone genes, such as pdi1 and bip1, were induced in response to increased production of cellulase proteins [5]. These data suggested that the intracellular secretory pathway tightly regulates lignocellulase secretion in particular components of the ER.

The ER acts as the central hub where membrane and secretory proteins are properly folded and matured. When the ER encounters high protein flux or intractable heterologous proteins, its folding capacity could be transiently saturated, thus leading to a secretory pathway traffic jam and causing ER stress [6,7]. Cells activate multiple pathways to respond to ER stress, one of the best characterized being the unfolded protein response 
(UPR). In Saccharomyces cerevisiae and filamentous fungi, the UPR mainly depends on an evolutionarily conserved signaling cascade which is mediated by ERresident transmembrane kinase/endoribonuclease IRE1 (Inositol-requiring enzyme-1) and the basic-leucine zipper (bZIP) transcription factor HAC1 (homologous to ATF/ CREB1) [8]. Although several aspects of ER stress response and the UPR have been intensively unraveled using the $S$. cerevisiae model, filamentous fungi are capable of much higher protein production levels, suggesting different or additional response mechanisms might exist between the two systems. For instance, filamentous fungi utilize a feedback mechanism termed repression under secretion stress (RESS) [9-11] which selectively downregulates transcription of genes encoding extracellular enzymes upon ER stress and thus helps to reduce ER load. However, the detailed mechanism behind RESS is not clear at the moment.

The Ascomycete Neurospora crassa is a common inhabitant of burnt plant material in nature and has recently become a model system to address lignocellulose deconstruction and utilization [12,13]. Previous transcriptome profiling of $N$. crassa grown on Miscanthus and cellulose revealed that the expression of most lignocellulase genes is significantly induced at early time points $(16 \mathrm{~h})$ but rapidly declines thereafter [14], implying RESS exists in $N$. crassa and might be a limiting step of lignocellulase synthesis. Moreover, recent work reported hac-1 activation by unconventional mRNA splicing when $N$. crassa was grown on cellulose [15], suggesting the UPR cascade is needed to coordinate the increased lignocellulase flux and the cellular folding capacity. N. crassa has the unique advantage of a nearly complete genome deletion strain collection [16], which makes it an ideal resource for a systematic study of the ER stress response and lignocellulase synthesis. To our knowledge, studies on the cross-talk of these two processes have not been performed so far.

In this work, by using high-throughput mRNA sequencing technology (RNA-Seq) coupled to genetic screens, we explored the connection between fungal lignocellulase secretion and ER stress on a genome-wide level. We thus defined the regulon of the ER stress response and identified dozens of genes as well as three novel transcription factors: RES-1 (NCU03699), RES-2 (NCU02724), and RRG-2 (NCU02413) to be involved in this integrated process.

\section{Results}

The experimental design setup for the analysis of mild and acute ER stress during cellulase production in $N$. crassa

To trigger ER stress while eliminating any non-specific drug response noise, the $N$. crassa wild-type (WT; FGSC\#2489, Mat A) was treated with two kinds of ER stress-causing agents: either dithiothreitol (DTT; prevents disulfide bond formation,) or tunicamycin (TM; inhibits $N$-linked glycosylation). Cells can dynamically activate distinct physiological processes, such as adaptation, alarm, or apoptosis, depending on the ER stress intensity $[7,17]$. In order to maximize the detection of targets which contribute to ER stress resistance and minimize apoptotic effects, three experiments were initially employed to determine the appropriate treatment (Figure 1). When the sensitivity of $N$. crassa WT to DTT and TM was measured using concentration gradients (Figure 1A), we observed a range of growth phenotypes from mild inhibition at low concentrations ( 1 to $3 \mathrm{mM}$ DTT or 0.5 to $10 \mu \mathrm{g} / \mathrm{mL} \mathrm{TM}$ ), to significant impairment at moderate concentrations (5 to $11 \mathrm{mM}$ DTT or 20 to $80 \mu \mathrm{g} / \mathrm{mL} \mathrm{TM}$ ) and complete inability to grow when the conidia were exposed to $13 \mathrm{mM}$ DTT or $120 \mu \mathrm{g} / \mathrm{mL} \mathrm{TM}$, respectively. We considered these results to reflect lethal, acute, and mild stress levels. We next checked the IRE-1-mediated mRNA splicing of the unfolded protein response marker hac-1 [15] by treating $N$. crassa cells with a similar concentration gradient of either DTT or TM during growth on cellulose. The results shown in Figure 1B suggest that a treatment with $0.1 \mathrm{mM}$ DTT or $5 \mu \mathrm{g} / \mathrm{mL}$ TM is sufficient to achieve mild stress levels. Finally, to determine the impact of distinct stress levels on lignocellulase synthesis, we measured the expression levels of two well-known UPR maker genes, grp-78 (NCU03928) and pdi-1 (NCU09223), as well as two major cellobiohydrolase genes, $c b h-1$ (NCU07340) and cbh-2(NCU09680), by quantitative PCR (qPCR) (Figure 1C). We found the folding machinery to be activated at all tested stress levels, while $c b h-1$ and $c b h-2$ were dramatically down-regulated at concentrations exceeding $0.1 \mathrm{mM}$ DTT or $5 \mu \mathrm{g} / \mathrm{mL} \mathrm{TM}$, respectively, suggesting an induction of RESS to render cells more resistant against acute ER stress. Thus, based on growth phenotype, the splicing pattern of hac-1 mRNA and RESS mediated down-regulation of cellulase genes, four stress conditions were finally chosen for further analysis: mild ER stress by treatment with $0.1 \mathrm{mM}$ DTT or $5 \mu \mathrm{g} / \mathrm{mL} \mathrm{TM}$; and acute ER stress by treatment with $5 \mathrm{mM}$ DTT or $40 \mu \mathrm{g} / \mathrm{mL}$ TM.

\section{Defining the ER stress response targets in wild-type $N$. crassa during cellulase production}

Comparative analyses of the transcriptional profiling data of WT under four ER stress conditions (mild ER stress and acute ER stress by treatment with DTT or TM as described above) versus the no drug condition were performed. Of the 9,730 annotated genes in the $N$. crassa genome, we detected 1,322 (13.59\%), 1,349 (13.86\%), $1,810(18.60 \%)$, and $1,882(19.34 \%)$ genes with altered expression which determined by NOISeq (for detail of cut-off, 


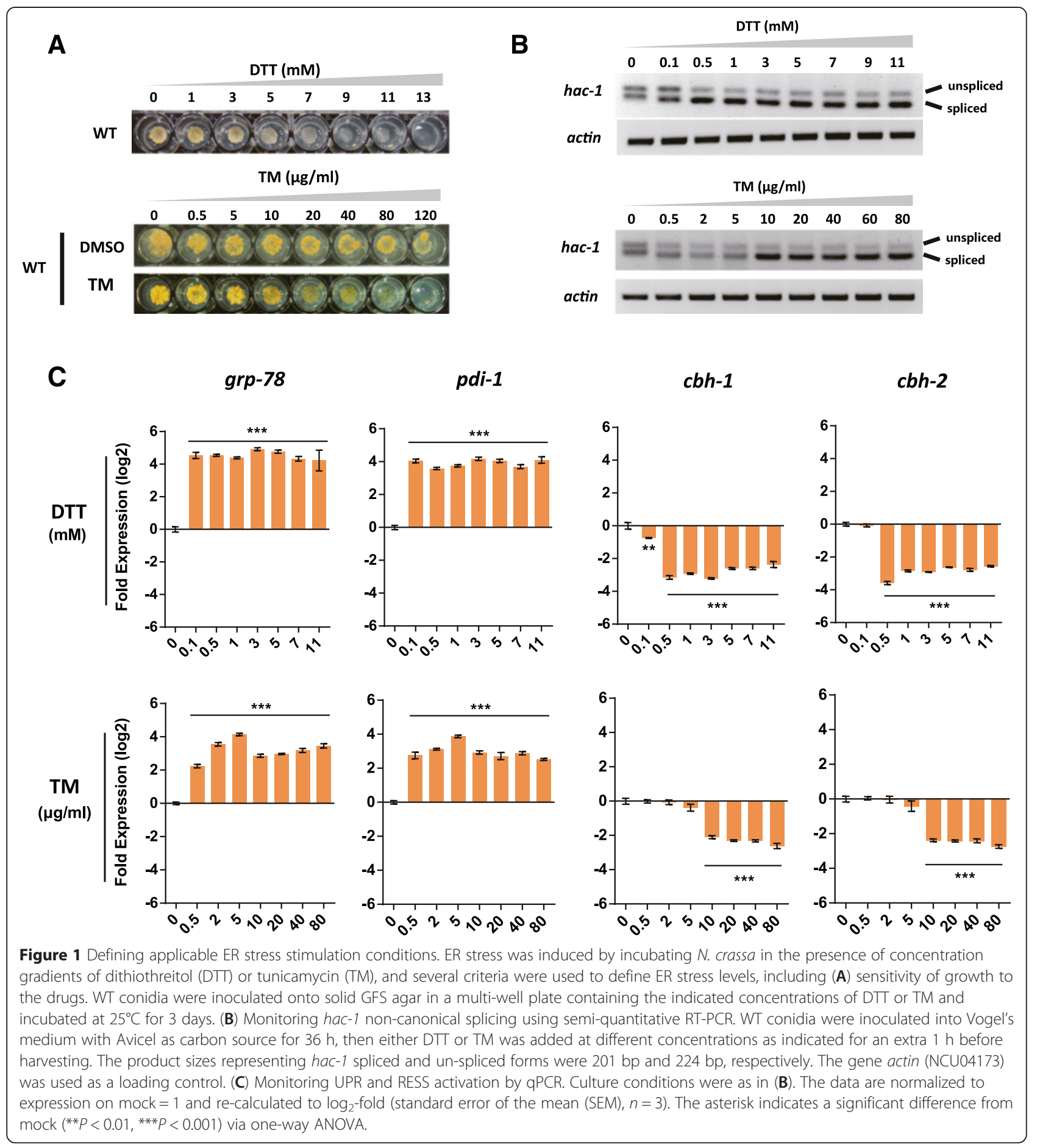

see the 'Materials and methods' section) for each of the four conditions, respectively (Additional file 1: Table S1 p1 and Additional file 2: Table S2 p1-4). The global regulation patterns during mild and acute ER stresses were graphed separately (Figure 2A). Elevated expression levels in a subset of $766(7.87 \%)$ genes induced by both DTT and TM at either mild or acute ER stress levels were deemed to be the result of true ER stress rather than non-specific effects and therefore defined as the ER stress response targets (ESRTs: 277 mild-specific targets, 285 acute-specific targets, and 204 genes generally up-regulated under both types of stress) (Figure 2B, Additional file 3: Table S3 p1, p3). Several wellestablished UPR targets [18] were found to be included in our ESRT dataset (Figure 2A). This result was confirmed 


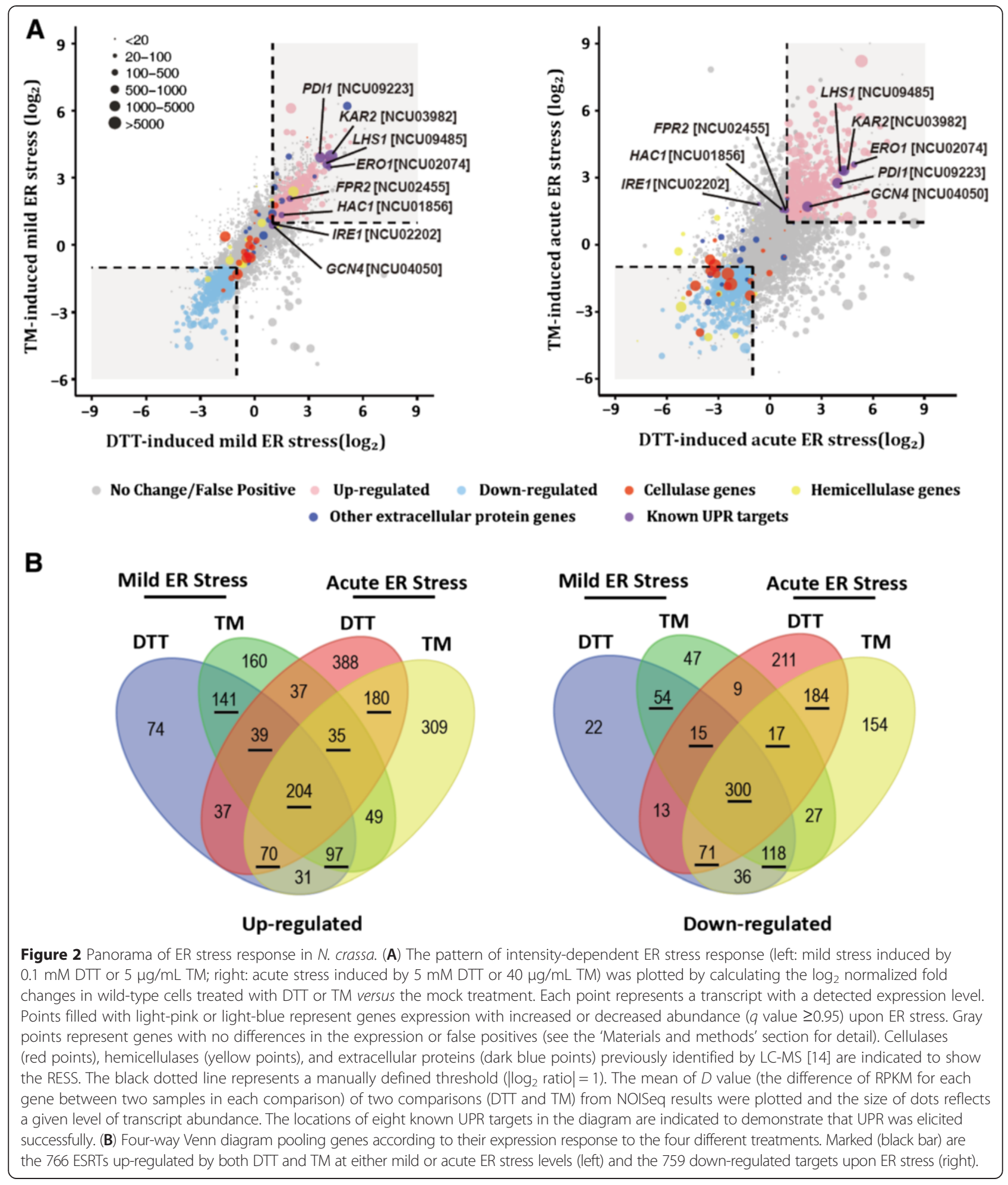

by qPCR assays using independent biological samples, showing consistency with the RNA-seq data (Additional file 4: Figure S1). Furthermore, we also collected 759 (7.80\%) genes (Figure 2B) with decreased abundance upon ER stress (Figure 2B and Additional file 3: Table S3 p4, p6).
Including cellobiohydrolase-1 (cbh-1, NCU07340) and cellobiohydrolase-2 (cbh-2, NCU09680), a total of 23 major lignocellulase genes were found to belong to this dataset and be specifically down-regulated under acute ER stress (Figure 2A and Additional file 3: Table S3 p7), 
implying that a rapid correction of intracellular transcript pools is needed to tolerate acute stress.

Further functional categorization of 462 (60.31\%) annotated genes of the 766 ESRTs using FunCat [19] in addition to manual classification based on Pfam domain annotations and BLAST hits showed most of them were linked to protein secretion or the biogenesis of secretory organelles (Additional file 3: Table S3 p2, p3). Overrepresented categories found were for example as follows: protein folding, modification, destination (155 genes, $P=1.49$ e-07), membrane lipid metabolism (19 genes, $P=3.89 \mathrm{e}-03)$, transport facilities and transport routes (166 genes, $P=1.86 \mathrm{e}-09$ ), as well as cell growth $/$ morphogenesis (41 genes, $P=4.52 \mathrm{e}-03$ ). Extending previous observations, the expression pattern of certain functional gene groups were found to depend on stress intensity. For example, genes encoding members of the p24 family (for example, NCU01342 [ERV25 in $S$. cerevisiae], NCU03800 [ERP3], and NCU04003 [ERP1]), which are involved in ER to Golgi transport, were specifically up-regulated upon mild stress levels. In contrast, genes encoding most components of COPII transport vesicles involved in ER to Golgi transport were generally up-regulated under any stress levels. In addition, the expression levels of half of the genes engaged in $\mathrm{N}$ linked or O-linked glycosylation (13 of 26) were enhanced solely under mild stress conditions whereas most genes related to phospholipid metabolism (9 of 13) were only upregulated upon acute stress intensity (Additional file 3: Table S3 p3). Furthermore, our ESRT dataset included several complete complexes within the secretory pathway, such as the SEC61 translocon complex (NCU08897 [SEC61 in S. cerevisiae], NCU08379 [SBH1/SBH2], and NCU04127 [SSS1]), the SEC63 complex (NCU00169 [SEC63], NCU06333 [SEC62], NCU02681 [SEC66], and NCU07746 [SEC72]), as well as the SNARE complex involved in vesicle-mediated Golgi to ER retrograde trafficking (NCU06708 [SEC22], NCU00953 [SEC20], NCU07939 [UFE1], and NCU00184 [USE1]). Given that around $40 \%$ of genes in the $N$. crassa genome are without clear function, the ESRTs identified here will aid in detecting novel component function in secretory pathway of filamentous fungi.

\section{Genome-wide overview of the ESRT deletion mutants in N. crassa}

To provide a basic understanding of the function of ESRT genes discovered by transcriptome analysis, we phenotypically screened 527 mutants of the total 766 ESRT targets (including 526 homokaryotic gene knockout strains and one single-base-pair deletion mutant for $c p c-1$ which results in a nonfunctional, truncated polypeptide of this amino acid regulator [20]) and 249 genes that appear to be pivotal for ER stress resistance in the
WT (Figure 3) for their sensitivity to ER stress (Additional file 5: Table S4). Most mutants were able to survive in the presence of $1 \mathrm{mM}$ DTT, except for strain $\triangle N C U 10762$. In S. cerevisiae, the homolog of NCU10762 is ALG7, which encodes the UDP-N-acetyl-glucosamine-1-P transferase (GPT) that transfers the first $\mathrm{N}$-acetylglucosamine to dolichyl-phosphate in the first step of the dolichol pathway of $N$-linked protein glycosylation [21]. Moreover, we found another 248 genes whose deletion caused the $N$. crassa cells to become DTT-sensitive, with 44, 84, 82, and 38 of them sensitive to $3,5,7$, and 9 mM DTT, respectively. As anticipated, many of these genes had roles in the secretory pathway, such as genes involved in protein translocation into the ER (for example, NCU02681 [SEC66 in S. cerevisiae], NCU08379 [SEC61], protein folding (for example, NCU00813 [MPD1], NCU09485 [LHS1], NCU 11102 [SCJ1], NCU00968 [SIL1]), ER-associated degradation (ERAD) (for example, NCU00146 [DER1], NCU 01296 [DER1], NCU01268 [UBC6]), protein glycosylation (for example, NCU06386 [ALG5], NCU06166 [KTR7]), and protein trafficking (for example, NCU03819 [SEC16], NCU 01342 [ERV25], NCU05514 [YIP1], NCU06708 [SEC22]). However, many mutants for genes with pivotal functions as unfolded protein response targets could not be assayed because they lacked homokaryotic mutants, such as grp-78 (NCU03982) and pdi-1 (NCU09223), as well as nearly half of the genes engaged in protein translocation, glycosylation, and trafficking (Additional file 3: Table S3 p3). It is likely that these genes are essential for cell viability.

Importantly, our screen provided functional data that $100(13.05 \%)$ formerly unclassified genes could be directly or indirectly involved in ER stress. To test whether these ESRTs also contribute to cellulase secretion, we next checked the cellulase production of 18 mutants sensitive to $3 \mathrm{mM}$ DTT and 31 mutants with sensitivity to $5 \mathrm{mM}$ DTT in liquid batch culture. Deletion of five genes, namely NCU07135, NCU08986, NCU04360, NCU01886, and NCU00918, decreased cellulase secretion titers by more than $30 \%$ when compared with the WT (Additional file 4: Figure S2), suggesting the products of those five genes might function in the secretory pathway. The function of the other candidates remains unknown, although the DTT-sensitive phenotype suggests they might be involved in the ER stress response.

\section{Identification of ER stress response targets that are regulated by the canonical IRE-1/HAC-1 UPR pathway}

As in S. cerevisiae, no clear homologs to ER stress sensors such as PKR-like ER kinase (PERK) and activating transcription factor-6 (ATF6) were found in N. crassa, implying the IRE-1 (encoded by NCU02202) and HAC-1 (NCU01856) mediated pathway might be the only route to trigger the UPR cascade. In comparison to the homologs from other species [22-24], the N. crassa hac-1 

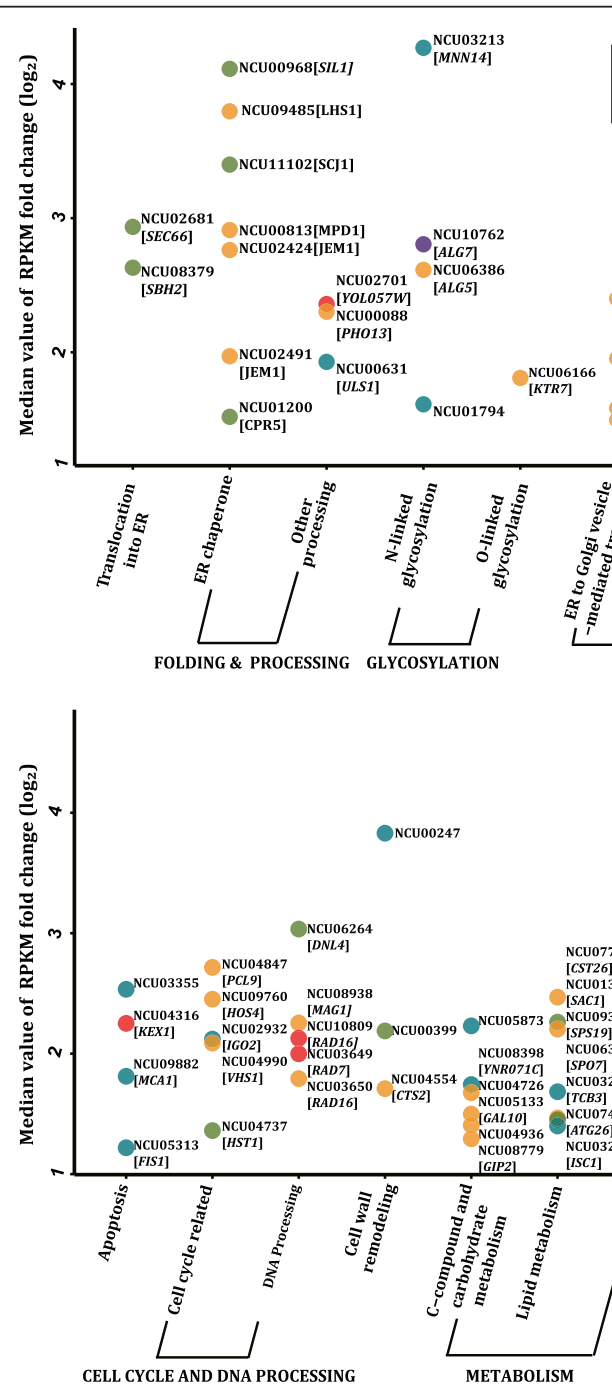

CELL CYCLE AND DNA PROCESSING

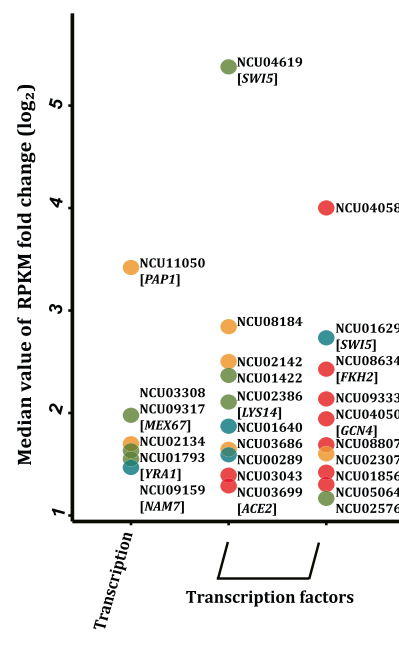

Figure 3 (See legend on next page.) [YPLO67C]

Mutants sensitive to $1 \mathrm{mM}$ DTT Mutants sensitive to $5 \mathrm{mM}$ DTT Mutants sensitive to $9 \mathrm{mM}$ DTT

Mutants sensitive to $3 \mathrm{mM}$ DTT Mutants sensitive to $7 \mathrm{mM}$ DTT

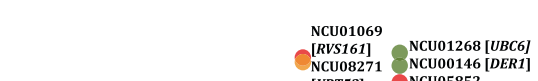

NCU08271 NCU00146 [DER $]$

NCU03819
[SEC16]
NCUNS1637
[RVI67]

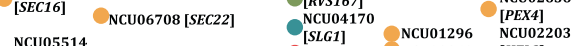

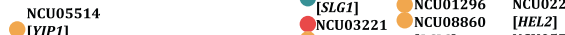

$\begin{array}{llll}{[M V P 1]} & \text { NCCL2] } & \text { NCU05777 } & \text { NCU04192 [APE1] }\end{array}$

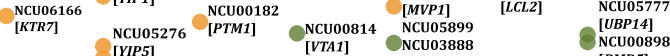

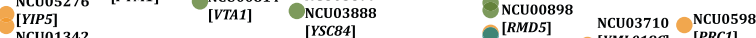
[ERV25]
[ECU04119[TLG2]

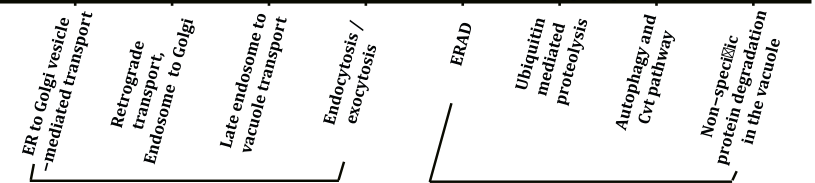

PROTEIN TRAFFICKING

$\mathrm{O}_{[P T H 1]}^{\text {NCU01070 }}$

$\mathrm{O}_{[B C S 1]}^{\text {NCU03921 }}$

ONCU04218 [RSB1]

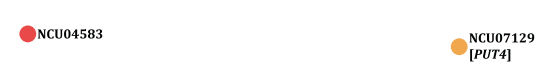

NCu07129
$[$ PUT4]

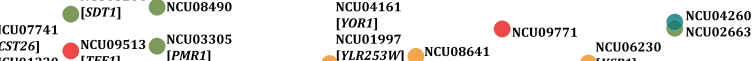

NCOO0290

[KSP1] NCU04924

ONCU09101 NCU03714 [TRX3]

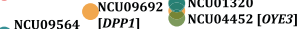

NCU03989 NCU0 ${ }_{\text {NCU1833 }}^{\text {NCU0712 }}$

[PHO88] NCU02885 [MKK1]

NCU03783 NCU07739 [YTP1]

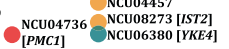

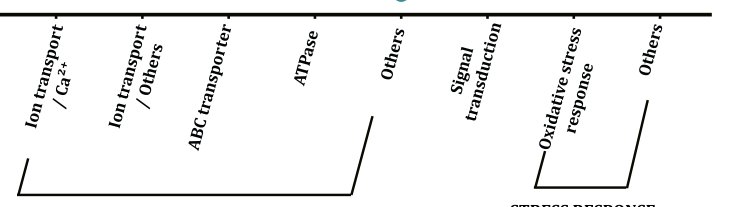

SUBSTRATES TRANSPORT

STRESS RESPONSE

Ncu04641

NCu09402 ONCU00868
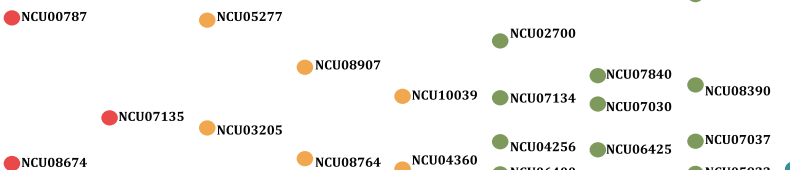

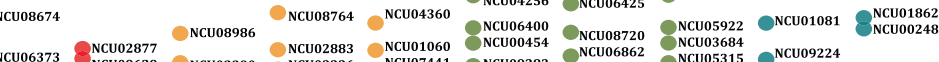

NCU09629
NCU02390 NCU06763 NCO3356 ONCU06926 NCU04544
NCU03773

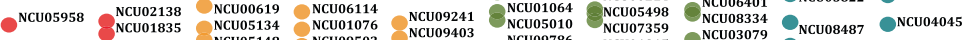

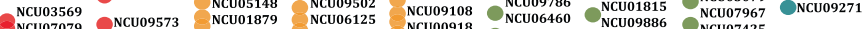

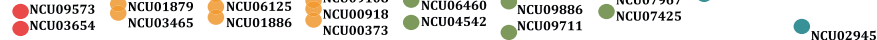

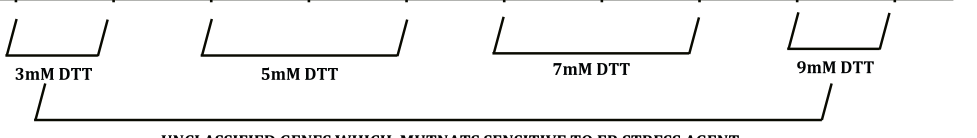

UNCLASSIFIED GENES WHICH MUTNATS SENSITIVE TO ER STRESS AGEN 
(See figure on previous page.)

Figure 3 Expression induction and functional categorization of 249 N. crassa KO mutants which exhibited ER stress susceptibility. Each point on the scatter plot represents the median value of fold changes of WT on mild DTT, mild TM, acute DTT, and acute TM (log 2 normalized, the full data see Additional file 3: Table S3, p3) and functional category of an ESRT gene whose KO mutants exhibited ER stress susceptibility. DTT-sensitive phenotypes are plotted using different colors, from strongest to weakest along a gradient of dark-purple (sensitive to $1 \mathrm{mM}$ DTT), red (3 mM), orange (5 mM), green (7 $\mathrm{mM})$ to cyan $(9 \mathrm{mM})$.

mRNA contains an atypical intron (23 nt) [15] similar in size to higher eukaryotes, such as Arabidopsis thaliana (23 nt), Drosophila melanogaster (23 nt), and Homo sapiens (26 nt), rather than $S$. cerevisiae (252 nt) (Additional file 4: Figure S3A), revealing an intimate phylogenetic link between higher eukaryotes and filamentous fungi. To explore how the IRE-1/HAC-1 mediated UPR pathway affects ER stress response and lignocellulase production, a KO mutant for hac-1 was constructed while Dire-1 was purified by sexual crossing (Additional file 4: Figure S3B,C,D). Consistent with previous observations in Aspergillus fumigatus [25] and other eukaryotes [22,23], a loss of IRE-1 was sufficient to impair the unconventional splicing of hac-1 mRNA in N. crassa (Additional file 4: Figure S3E). In comparison to their parental strains, both $\Delta i r e-1$ and $\Delta h a c-1$ mutants showed a marked reduction (approximately 32\%) in radial growth on solid minimal medium (Figure 4A). Deletion of either ire-1 or hac-1 did not visibly affect the width (approximately $5 \mu \mathrm{m}$ ) of the middle intercalary cell of conidia in $N$. crassa (Additional file 4: Figure S4A); however, during germination, huge vacuoles accumulated within young hyphae of both mutants, probably caused by a vesicle trafficking malfunction (Additional file 4: Figure S4B). In liquid batch culture using microcrystalline cellulose (Avicel) as the sole carbon source, more than $97 \%$ of both $\Delta$ ire- 1 and $\Delta$ hac- 1 conidia failed to germinate (Figure $4 \mathrm{~B}$ ), suggesting UPR is a key factor for the cellulase secretion capacity of N. crassa.

We further explored which ESRTs were regulated by the UPR pathway through transcriptome profiling of both $\Delta$ ire- 1 and $\Delta h a c-1$ strains (Additional file 1: Table S1 p2 and Additional file 2: Table S2 p5-10). Because these strains are unable to use cellulose, sucrose pre-grown mycelia were used for the profiling analysis instead of directly inoculating cultures with conidia. Bioinformatic analysis (Additional file 6: Supporting information) revealed that 223 and 186 genes out of the 766 ESRTs were regulated by IRE-1 and HAC-1, respectively (Additional file 7: Table S5 p5). As expected from previous observations in S. cerevisiae [18,26] and other model systems [11,25,27-29], the IRE-1/HAC-1 governed UPR regulon mainly functions to facilitate protein folding and to remodel the secretory pathway. Major enriched functional categories (FunCat) were protein folding and stabilization $(P=4.44 \mathrm{e}-06$ for IRE-1, $P=3.72 \mathrm{e}-06$ for HAC- 1$)$, glycosylation $(P=1.15 \mathrm{e}-$

A

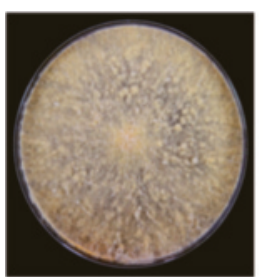

FGSC2489

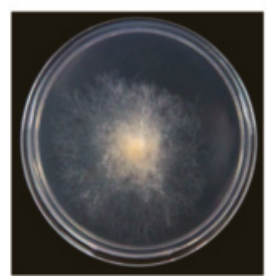

Dire-1

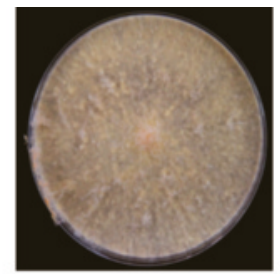

FGSC9720

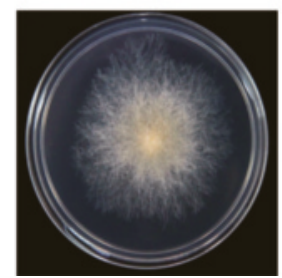

Dhac-1

B

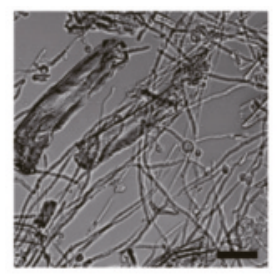

FGSC2489

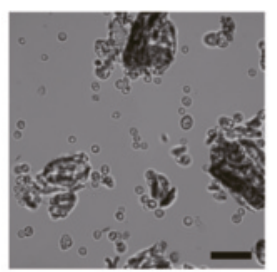

Dire-1

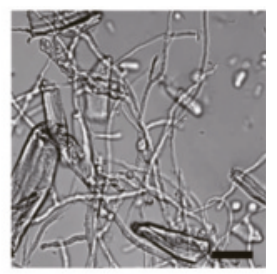

FGSC9720

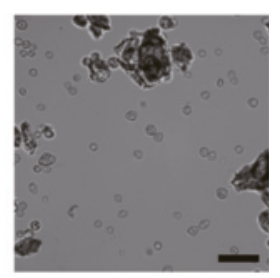

Dhac-1

Figure 4 Deletion of ire-1 and hac-1 affects cell growth and causes cellulose utilization defects. (A) Comparison of radial growth phenotypes of ire-1 and hac- 1 with their parental strain. Conidia (around $10^{3}$ ) were dotted on minimal medium (MM) plates, grown for $72 \mathrm{~h}$ at $25^{\circ} \mathrm{C}$. (B) The conidia of $\Delta i r e-1$ and $\Delta$ hac-1 mutants fail to germinate under cellulolytic induction conditions (presence of Avicel as sole C-source). Pictures were taken after $36 \mathrm{~h}$ of culture; the irregular blocks in the images represent Avicel particles. The black bar indicates a length of $20 \mu \mathrm{m}$. 
11 for IRE-1, $P=7.07 \mathrm{e}-13$ for HAC-1) as well as vesicular transport (Golgi network, etc.) $(P=6.35 \mathrm{e}-24$ for IRE-1, $P=5.11 \mathrm{e}-22$ for HAC-1). Although $\Delta$ ire-1 and $\Delta$ hac-1 failed to grow on Avicel, lignocellulase genes were found to be induced to similar levels compared with their parental strains (Additional file 1: Table S1 p2), suggesting the UPR pathway does not directly regulate the induction of hydrolytic enzyme genes by cellulose. In addition, the expression levels for most lignocellulase genes were markedly down-regulated when acute ER stress emerged in both the $\Delta$ ire-1 and $\Delta$ hac- 1 backgrounds (Additional file 1: Table S1 p2 and Additional file 2: Table S2 p6, p8), implying RESS is independent of the UPR pathway. Furthermore, the analysis of intracellular cellulase synthesis and secretory pathway protein loading in $\Delta i r e-1$ and $\Delta h a c-1$ revealed that the cellulase synthesis in both mutants was similar to the parental strains (after a culture switch from sucrose to cellulose for $4 \mathrm{~h}$ and $36 \mathrm{~h}$ ). Therefore, the observed reduction in secreted cellulases implies a reduced secretion capacity within this time frame. At a later stage ( $96 \mathrm{~h}$ ), the amount of intracellular cellulases was diminished, which might be a result from a negative feedback-loop of cellulase hypo-secretion (Additional file 4: Figure S5A,B). These observations demonstrate that IRE-1 and HAC-1 play an important role in the $N$. crassa cellulase secretion system.

\section{Systematic characterization of TFs required for ER stress response during cellulase synthesis}

For both ER stress response and cellulase synthesis, transcription factor (TF) mediated orchestration of gene expression is crucial. We systematically searched for TFs with significantly altered expression patterns in our recorded differential expression data based on acute/mild ER stress (Figure 2). Of the 266 predicted TFs present in the $N$. crassa genome (based on Broad version 7) [30] (Additional file 8: Table S6 p1-2), 33 were up-regulated in the presence of ER stress, while 6 TFs were downregulated (Figure 5A and Additional file 8: Table S6 p3).

Of the 33 TFs with increased abundance upon ER stress, five (15.15\%) belonged to the basic-region leucine zipper (bZIP) family, 11 (33.33\%) to the fungal $\mathrm{Zn}(2)$ Cys(6) binuclear cluster family, nine (27.27\%) to the Cys2His2 (C2H2)-type zinc fingers, and eight to miscellaneous families (24.24\%) (Additional file 8: Table S6 p3). We next screened 30 available homokaryotic deletion mutants out of $33 \mathrm{TFs}$ for DTT-sensitivity; compared with WT, 20 TFs exhibited significant DTT-sensitive phenotypes, including the UPR regulator hac-1 (NCU01856) (Figure 5B). Additionally, six TFs were found to be regulated in an IRE-1/HAC-1 mediated manner; including four TFs (NCU09333, NCU03043, NCU03686, NCU02386) affected by IRE-1 and two TFs (NCU01422, NCU06095) that were dependent on HAC-1 (Additional file 7: Table S5 p5).
To assess whether those TFs regulated by ER stress also contribute to cellulase secretion, liquid batch cultures with (Figure 5C) or without (Additional file 4: Figure S6) a rich nitrogen source were performed. The Aire-1 and $\Delta$ hac-1 strains, along with their parental strains, were included as controls. Consistent with previous observations [31], the $\Delta c r e-1$ strain displayed an increase of approximately $50 \%$ in the amount of secreted protein compared to the WT strain. A loss of ire-1 led to a $50 \%$ decrease of the secreted protein levels in comparison to the WT strain, while the $\Delta$ hac-1 KO mutant almost completely lost its ability to produce lignocellulases. Using $\pm 30 \%$ of secreted proteins produced by WT as a threshold (refer to the $\Delta c r e-1$ published data [31]) and considering the data from both nitrogen conditions, we found $\triangle N C U 03699$ could significantly promote lignocellulase secretion similar to $\triangle c r e-1$, while $\triangle N C U 02413$ $(\Delta r r g-2)$ and $\triangle N C U 02724$ exhibited repressive effects. The intracellular cellulase loading of the secretory pathway was further examined in these mutants using switch experiments of sucrose pre-grown strains shifted to Avicel. While the intracellular cellulase production was not affected until after 96 h (Additional file 4: Figure S5A,B), extracellular cellulase accumulation was substantially increased ( $\triangle N C U 03699)$ or reduced $(\triangle N C U 02413(\Delta r r g-2)$ and $\triangle N C U 02724$ ) (Additional file 4: Figure S7A,B), implying that the secretion capacity rather than cellulase synthesis is affected in these strains. These findings suggest that the hyper- or hypo-secretion phenotype of these mutants is linked to cellulase secretion (Additional file 4: Figure S7A,B). To the best of our knowledge, this is the first report providing strong functional data indicating that these TFs are involved in lignocellulase production and ER stress, although NCU02413 ( $r r g-2)$ had previously been suggested to play a role in the oxidative stress response in N. crassa [32]. Based on their involvement in the overall process, we named the TFs encoded by NCU03699 and NCU02724 RES-1 and regulator for ER stress response (RES-2), respectively.

Of the six TFs with decreased abundance upon ER stress, three belong to the fungal $\mathrm{Zn}(2)-\mathrm{Cys}(6)$ binuclear cluster domain family, including the two core regulators of plant cell wall degradation: CLR-2 [33] and XLR-1 [34]. Because most lignocellulase genes that are down-regulated in the presence of acute ER stress belong to the CLR-2 or XLR-1 regulons, it seems likely that RESS results to a great extent from the down-regulation of these core regulators. Two of the other four TFs that were found to be down-regulated under ER stress (NCU09549, NCU04848, NCU08923, and NCU07430 had available mutants that allowed us to test their enzyme production. Slightly decreased lignocellulase production was recorded for $\triangle \mathrm{NCU} 09549$ when compared to WT, while no obvious defect was observed in the $\triangle$ NCU04848 mutant (Figure 5C). 


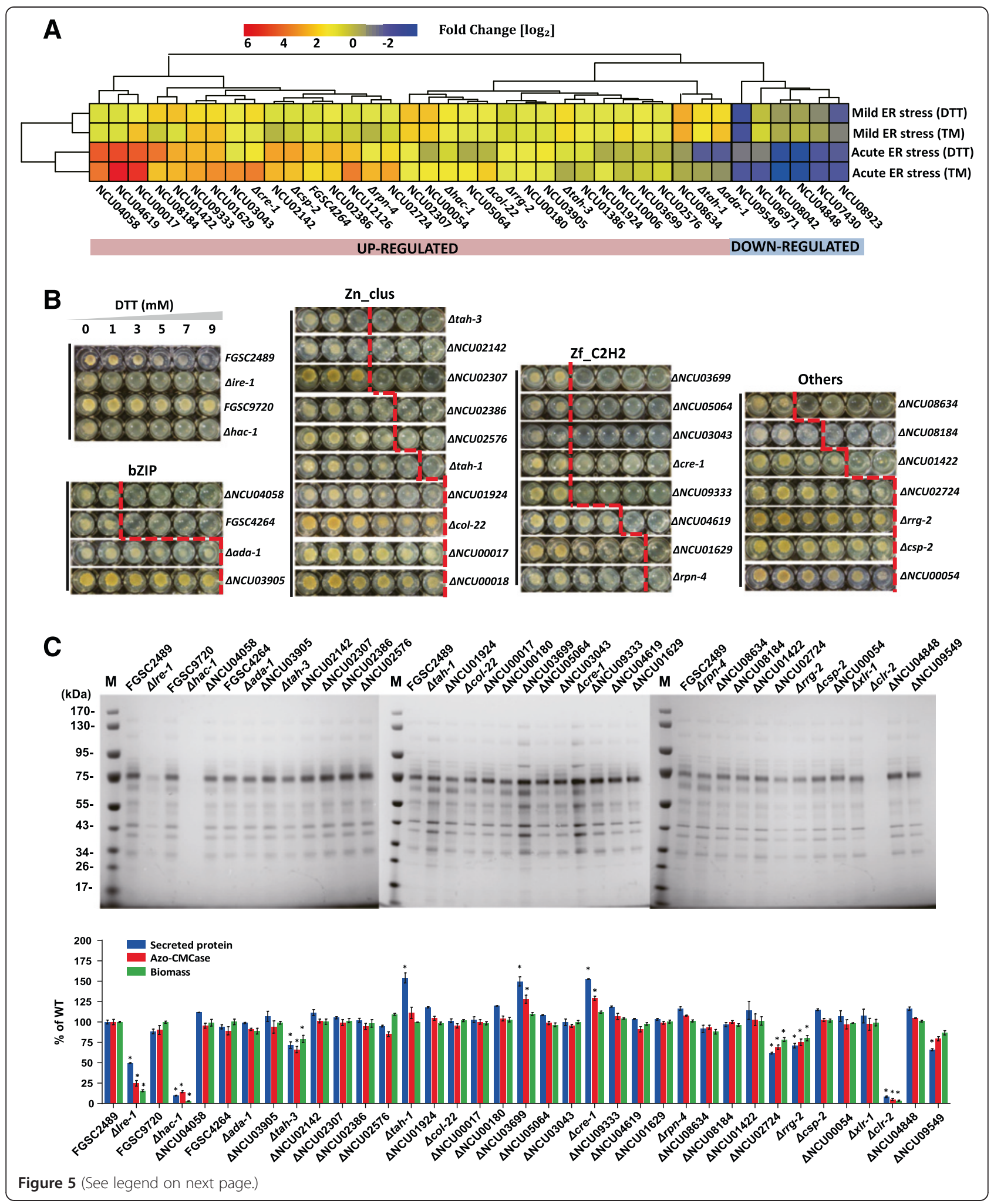


(See figure on previous page.)

Figure 5 Screen for transcription factors involved in ER stress response and cellulase synthesis. (A) Heatmap of 39 transcription factors (TFs) found to be differentially expressed in response to ER stress under distinct stress intensities: 33 up-regulated and 6 down-regulated. The degree of expression abundance was normalized by RPKM-fold change $\left(\log _{2}\right)$. Names of TF DNA-binding domains according to Pfam annotation were indicated in square brackets. (B) Screening of DTT susceptibility of 30 available ESRT TF KO mutants. The red line indicates the growth to no growth threshold and is an indicator for sensitivity to ER stress elicited by DTT. (C) Batch cultures of ESRT TF KO mutants were prepared to discover targets affecting cellulase secretion. After 7 days of culture with rich nitrogen source, the typical secretome of each TF candidate is shown on SDS-PAGE gel (upper panel), while total extracellular protein concentration, CMCase activity, and biomass (as total protein) were measured and displayed after normalization to the WT control (lower panel) by percentage (standard error of the mean (SEM), $n=3$, the asterisk $\left(^{*}\right.$ ) indicates a significant difference from WT with an unadjusted $P$ value of $<0.001$ using a one-way ANOVA).

RES-1, RES-2, and RRG-2 are required for ER stress response and cellulase synthesis

Since RES-1, RES-2, and RRG-2 likely function to bridge the ER stress response to lignocellulase expression, transcriptional profiling of the three corresponding TF deletion strains was performed under ER stress conditions. Deletion of res-1 (encoded by NCU03699) resulted in up-regulation of 583 genes in comparison to WT in cells grown on Avicel for $36 \mathrm{~h}$. Consistent with the protein secretion data (Figure 5C), FunCat analysis of the 583 potential res- 1 targets showed 38 genes belonging to the 'polysaccharide metabolism' group, with many lignocellulase genes being enriched $(P=6.11 \mathrm{e}-09)$ (Figure 6A and Additional file 9: Table S7 p1). For example, the gene expression of several $A A 9$ family members such as NCU08760 (AA9-5), NCU03328 (AA9-6), NCU00836 (AA9-7), NCU03000 (AA9-8) as well as some other glycosyl hydrolases such as NCU05121 (gh45-1, endoglucanase V), NCU06861 (gh43-3), NCU07326 (gh43-6), and NCU04952 (gh3-4, $\beta$-glucosidase) showed a significant increase in the $\Delta$ res- 1 strain compared to WT. The expression levels of NCU09664 and NCU04870, encoding acetylxylan esterases, increased more than 33- and 7fold, respectively, and a gene encoding the extracellular feruloyl esterase B (NCU09491) increased over 30-fold upon loss of RES-1. Surprisingly, the expression of
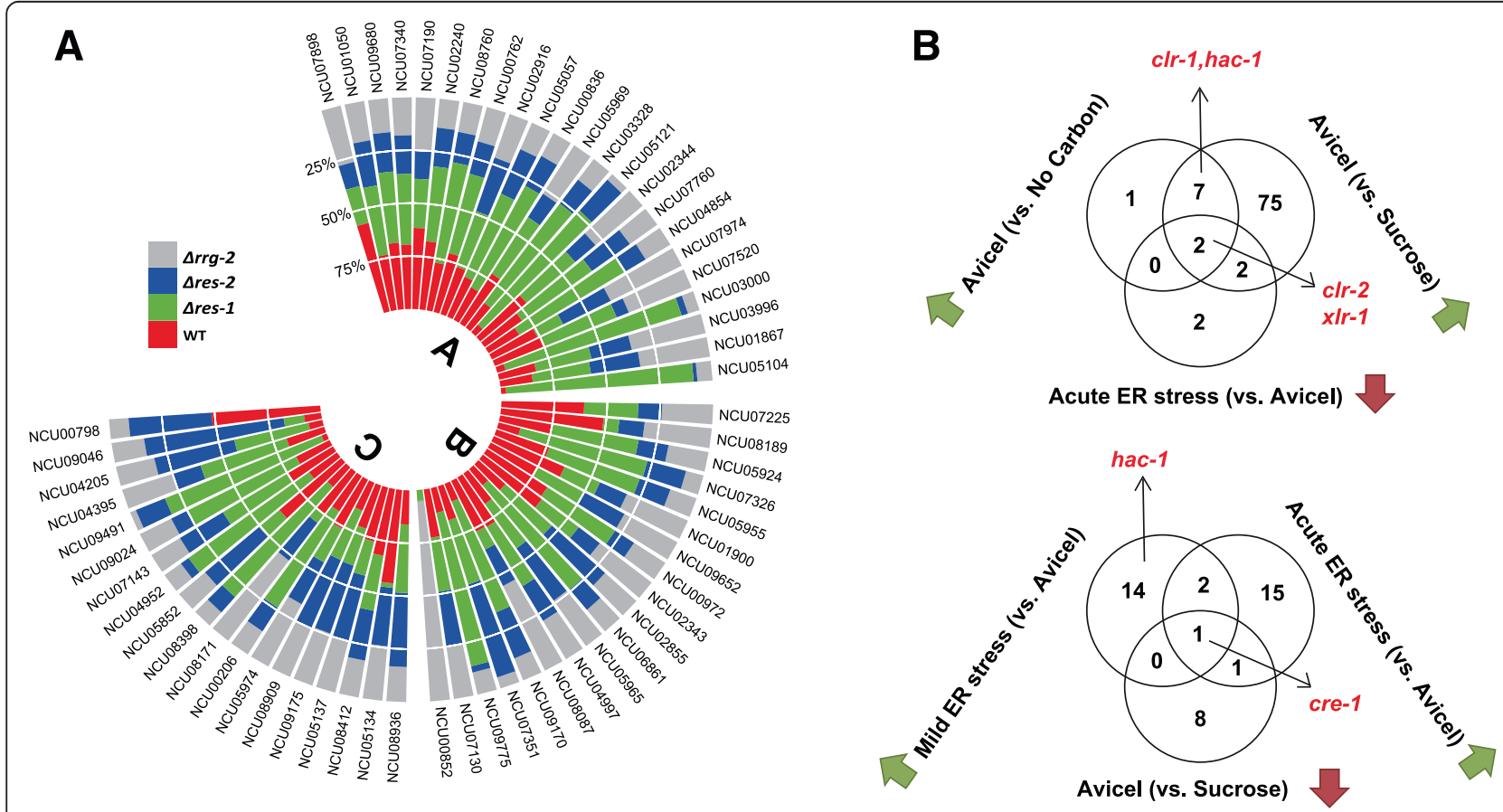

Figure 6 RES-1, RES-2, and RRG-2 indirectly affect lignocellulase gene expression. (A) Comparison of lignocellulase expression levels in $\Delta$ res-1, $\Delta r e s-2$, and $\Delta \mathrm{rrg}-2$ with WT. A total of 62 genes were grouped into three clusters: [A] cellulase genes, [B] hemicellulase genes, and [C] other genes encoding extracellular proteins. For each target, the relative proportion of transcript abundance (RPKM) is indicated as a percentage of the total. (B) Three-way Venn diagrams generated to search for TFs, which are coordinately expressed with lignocellulase genes. Top diagram: TFs induced (green arrow) by Avicel versus no carbon (top left) and versus sucrose condition (top right) and repressed (red arrow) under acute ER stress (bottom) were reasoned to potentially have a positive effect on lignocellulase gene expression. Bottom diagram: In addition, TFs being repressed when exposed to Avicel versus sucrose treatment while being induced upon ER stress could likely exert a negative effect on lignocellulase gene expression. 
several major cellulase genes, such as NCU07340 (cbh1), NCU09680 (cbh-2), and NCU00762 (gh5-1, endoglucanase 3), was WT-like in $\Delta$ res-1 after growth on Avicel for $36 \mathrm{~h}$, although significant hyper-secretion was observed after 7 days of culture (Figure 5C), suggesting RES-1 could affect lignocellulase production not only at the transcriptional but also at the post-transcriptional level as protein, thus the latter effect is exerted indirectly. In good agreement with this hypothesis, we found that a deletion of res-1 resulted in 76 ESRTs failing to be up-regulated upon exposure to DTT (Additional file 9: Table S7 p2-3), especially genes functioning in glycosylation $(P=2.8 \mathrm{e}-03$, for example, NCU02541 and NCU03995, which separately encode the oligosaccharyltransferase alpha and gamma subunit-like proteins) as well as lysosomal and vacuolar protein degradation $(P=5.38 \mathrm{e}-05$, for example, NCU01955 [autophagy-related protein 3], NCU02515 [dipeptidyl aminopeptidase]).

The $\Delta r e s-2$ and $\Delta r r g$-2 deletion strains exhibited repressive effects on lignocellulase secretion. Transcriptome profiles showed deletion of res-2 specifically downregulated 491 genes compared with WT when cells were grown on Avicel, including 87 genes involved in Ccompound and carbohydrate metabolism $(P=1.62 \mathrm{e}-06)$ (Figure 6A and Additional file 9: Table S7 p4). For instance, hemicellulase genes such as NCU07225 (gh11-2, endo-1, 4-beta-xylanase 2), NCU08189 (gh10-2, endo-1,4beta-xylanase), and NCU00972 (gh53-1, arabinogalactan endo-1,4- $\beta$-galactosidase) decreased more than three- to fourfold under Avicel conditions in the $\Delta$ res-2 mutant as compared to WT. We noticed the major cellulase genes such as NCU07340 (cbh-1), NCU09680 (cbh-2), and NCU00762 (gh5-1) also showed a $1.6 \sim 1.8$-fold decrease in expression under Avicel conditions in the $\Delta$ res-2 strain, although the respective data did not pass the $Q$ value cutoff. In addition, 118 ESRTs were shown to be regulated by the TF RES-2, including targets involved in cellular export and secretion $(P=7.68 \mathrm{e}-03)$, cellular import $(P=5.49 \mathrm{e}-03)$, enzyme inhibitors $(P=7.10 \mathrm{e}-03)$, as well as several targets functioning in autophagy and cytoplasm to vacuoletargeting (Cvt) pathways, such as NCU01955 (autophagyrelated protein 3), NCU04192 (vacuolar aspartyl aminopeptidase Lap4), and NCU01545 (autophagy protein 8) (Additional file 9: Table S7 p5-6).

Similar to res-2, a deletion of $r r g-2$ down-regulated 102 genes compared with WT when cells were grown on Avicel, including 25 genes involved in C-compound and carbohydrate metabolism $(P=6.11 \mathrm{e}-05)$ (Figure 6A and Additional file 9: Table S7 p7), such as lignocellulase genes NCU09680 (cbh2), NCU02240 (AA9-1, endoglucanase II), and NCU05955 (xyloglucanase). The expression of genes encoding glucoamylases was also found to be downregulated (for example NCU01517 and NCU08746). In addition, $r r g$ - 2 also appeared to regulate 115 ESRTs related to lipid, fatty acid, and isoprenoid metabolism $(P=4.90 \mathrm{e}-$
03), glycosylation $(P=1.97 \mathrm{e}-03)$, ER to Golgi transport ( $P=9.05 \mathrm{e}-03$ ), etc. (Additional file 9: Table S7 p8-9).

Lignocellulase genes can be induced significantly when Neurospora is exposed to cellulose; however, even in the presence of a cellulosic inducer, these genes can also be rapidly down-regulated when suffering RESS. Therefore, TFs with a similar expression pattern to lignocellulase genes under both physiological conditions are likely to be involved in cellulase regulation. We re-analyzed published transcriptome profiling data of $N$. crassa grown on sucrose, Avicel, and no carbon (starvation) and compared these with our ER stress response data [35] (Additional file 8: Table S6 p3-4; Figure 6B). Ten and 86 TFs were significantly induced by Avicel compared to either the no carbon or sucrose controls, respectively (Figure 6B, upper panel); nine of them were up-regulated in both conditions. Besides $c l r-1, c l r-2$, and $x l r-1$, hac-1 was also included, suggesting lignocellulase induction and UPR activation act synergistically. Filtering additionally for TFs with decreased abundance under acute ER stress (six total; potentially due to RESS), only $c l r-2$ and $x l r-1$ overlapped, implying CLR-2 and XLR-1 maybe the only direct activators of the lignocellulase regulon. Trying to find the key repressor of cellulase genes, we reasoned that such a regulator might be up-regulated under ER stress (mild as well as acute) and down-regulated under standard cellulolytic induction (Avicel only) compared to the repression condition on sucrose. Only cre-1 was identified to match these criteria (Figure 6B, lower panel) and is therefore confirmed to have a major influence on lignocellulase gene expression. However, this result does not imply that CRE-1 is the only repressor for cellulose genes, and additional repressors that are not necessarily co-regulated might still be involved. The expression pattern for res-1, res-2, and $r r g-2$ did not correlate with major cellulase genes or core regulators such as $c l r-2$, $x l r-1$, and cre-1, suggesting their influence on cellulase expression may occur through another way.

\section{Discussion}

In the present study, we systematically analyzed the ER stress response of $N$. crassa during lignocellulase induction and secretion by experiments at both mild and acute stress levels. We found 766 genes to be specifically up-regulated in response to this process (ESRTs), of which 223 and 186 genes were regulated by UPR pathway core components IRE-1 and HAC-1, respectively. Screening 527 deletion strains for ESRTs lead to the identification of 249 genes that appear to be pivotal for resistance to ER stress in the WT. Of these, 100 targets were previously annotated as 'unknown' and are reported here for the first time to be involved in the response to ER stress. Interestingly, we found 357 ESRTs to be highly conserved, with orthologs in higher eukaryotes such as human 
(Additional file 3: Table S3 p3). For example, the ESRT NCU09101 encodes a hypothetical protein and has orthologs in both $S$. cerevisiae (YBR220C) and $H$. sapiens (SLC33A1/AT-1). The function of S. cerevisiae YBR220C is unknown; however, $H$. sapiens SLC33A1/AT-1 encodes an ER membrane transporter that was recently reported to regulate the induction of autophagy downstream of the IRE1/XBP1 pathway [36]. Our screening data showed $\triangle N C U 09101$ was sensitive to DTT (Additional file 5: Table S4), consistent with previous reports, validating that this target is related to ER stress. To our knowledge, systematic information on ER stress at a cellular level has only been performed in three model systems to date, including screening of $\mathrm{KO}$ mutants in S. cerevisiae [37] and screening of natural variation of human B cells [38] and Drosophila lines [39]. Thus, our data not only fill the knowledge gap in filamentous fungi but also contribute to the general understanding of the mechanisms mediating this process in higher eukaryotes.

In this study, we further addressed how TF networks coordinate the signal flow and gene expression during the ER stress response and cellulase synthesis. Thirty-three TFs were up-regulated upon ER stress under conditions of cellulase synthesis, including the well-characterized UPR regulator HAC-1 (NCU01856) as well as CPC-1 (NCU04050). We found N. crassa HAC-1 to act as an important factor for lignocellulase secretion while not mediating the RESS feed-back loop (profiling data mentioned above are presented in Additional file 1: Table S1 p2). In addition, we found that hyphal growth is severely impaired in hac-1 deletion strains of N. crassa, which is consistent with previous observations in Aspergillus niger [40], suggesting that HAC-1 plays an important role in hyphal polarized growth and development, as it was shown in Candida albicans [28]. Interestingly, though the observed phenotype of $\Delta$ ire-1 was slightly weaker than the phenotype changes in $\Delta h a c-1$, both mutants showed a pronounced defect in their conidial germination and cellulase secretion capacity compared to their parental strains. Moreover, an un-spliced hac-1 transcript is present in the $N$. crassa $\Delta$ ire-1 strain. While in $S$. cerevisiae an un-spliced hac-1 mRNA cannot be translated into a functional protein [41], previous studies could show that mammalian cells are indeed capable of translating un-spliced $h a c-1 / X b p-1$ transcripts [42]. Whether such an event also occurs in filamentous fungi and in which way these proteins would contribute to certain physiological functions such as hyphal polarized growth remain unclear. The TF $\mathrm{CPC}-1$ has been previously characterized as a regulator associated with amino acid biosynthesis in N. crassa [43]. In metazoans, ATF4 as the functional homolog of $\mathrm{CPC}-1$ has been proven to act in the PERK mediated UPR branch and it regulates those UPR genes involved in redox response and apoptosis
$[6,7,11]$. The fact that $\mathrm{CPC}-1 / \mathrm{CpcA}$ is specifically induced upon ER stress has been observed in several independent fungal systems $[11,44,45]$ and could be confirmed by our data. Here, we found additionally that a deletion of $c p c-1$ renders $N$. crassa cells sensitive to DTT (Figure 5B) while significantly increasing extracellular protein secretion under limited nitrogen conditions (Figure S5). These observations suggest that $\mathrm{CPC}-1$ may play a critical role in $N$. crassa ER stress response and cellulase secretion, although its regulation does not seem to be controlled by the IRE-1/HAC-1 mediated UPR cascade (Additional file 7: Table S5 p5). With NCU04058, a novel putative bZIP TF was identified, whose expression levels were markedly elevated upon acute ER stress. Moreover, the fact that the corresponding deletion strain displayed an apparent DTT-sensitive phenotype implies that it might also function as a stress response regulator.

The TF genes res-1 (NCU03699) and NCU03043 are the homologs of $A$. fumigatus $z f p A$ and $f l b C$, respectively. It has been reported that both $A$. fumigatus $z f p A$ and $f l b C$ transcripts can be induced by exposing cells to calcium [46,47], suggesting RES-1 and the TF encoded by NCU03043 may respond to intracellular calcium disturbances induced by ER stress. Moreover, we saw that loss of RES-1 results in a boost of lignocellulase secretion, probably owing to the de-repressive effect of several auxiliary cellulose-degrading enzymes on transcription (for example, several AA9 family members) which contribute to promote the efficiency of classical hydrolytic enzymes (cellulases). Deletion of res-2 (NCU02724) and rrg-2 (NCU02413) on the other hand, significantly decreased lignocellulase expression and secretion in $N$. crassa, although probably in an indirect manner. In this work, FunCat characterization of the RES-2 regulon revealed that genes involved in cellular export and secretion $(P=7.68 \mathrm{e}-03)$ as well as cellular import (endocytosis) $(P=5.49 \mathrm{e}-03)$ were enriched. Moreover, endocytosis has been reported to contribute to hyphal tip growth and tip secretion [48], suggesting RES-2 might regulate secretory pathways of $N$. crassa. In addition, the ortholog of RES-2 in A. fumigates, Afu1g09670, has been reported to be induced by calcium treatment [46]. While ER stress is always associated with a disturbance in the intracellular calcium homeostasis, changes in calcium levels are also reported to influence the hyphal tip growth $[49,50]$ and thus might in turn affect tip secretion. Therefore, it is feasible, that RES-2 is involved in the ER stress response and affects lignocellulase secretion.

The $S$. cerevisiae SKN7, a homolog of the $N$. crassa RRG-2 (Identity: $57.5 \%, E$ value: $1 \mathrm{E}-38$ ), controls the oxidative stress response in yeast cells together with YAP1 [51]. It has been reported that over-expression of YAP1 contributes to modified intracellular redox conditions and enhances recombinant protein secretion [52]. However, whether SKN7 is also involved in protein secretion 
remains to be elucidated. In this work, deletion of RRG$2 /$ SKN7 in $N$. crassa leads to a hypo-secretion phenotype, suggesting that RRG-2/SKN7 maybe has similar functions as YAP1 in the protein secretion process. It is also known that ER stress and oxidative stress are closely linked events, and therefore antioxidants, which can diminish oxidative stress [53], could additionally decrease ER stress and improve protein secretion. Hence, it can be concluded that RRG-2 might be involved in the regulation of the redox balance to affect cellulase secretion. The TF TAH-3 (NCU03686) was previously found to be required for fungal tolerance to harsh non-thermal plasma treatment in $N$. crassa [54]. The counterpart of $\mathrm{TAH}-3$ in $S$. cerevisiae is UPC2, which functions by regulating sterol biosynthetic gene expression [55]. Potentially, disturbed sterol biosynthesis could trigger ER stress [56]. We found that extracellular protein secretion was reduced by circa $30 \%$ in $\Delta$ tah-3 compared to the WT, suggesting TAH-3 might be involved in ER stress resistance by maintaining intracellular sterol homeostasis.

The TF encoded by NCU09333 was identified as the homolog of the cellulase repressor ACE1 in T. reesei [57] and the stress responsive factor StzA in Aspergillus nidulans [58]. In N. crassa, NCU09333 was up-regulated upon ER stress while showing only a limited effect on cellulase secretion, suggesting NCU09333 functions more like StzA rather than ACE1 in N. crassa.
Finally, the expression level of the carbon catabolite repression (CCR) regulator CRE-1 (NCU08807) was found to be up-regulated upon ER stress. The $\Delta c r e-1$ strain moreover showed a DTT-sensitive phenotype, suggesting the CCR pathway might also cross-talk with the ER stress response.

By monitoring TFs with a decreased abundance upon ER stress, we detected CLR-2 and XLR-1. This result was confirmed by qPCR (Additional file 4: Figure S8), suggesting that the RESS-mediated repression of cellulase genes was likely a direct result of the down-regulation of these two regulators. The expression levels of some carbohydrate transporter genes, which are belonging to the $\mathrm{clr}$ 2 and $x l r-1$ regulons, such as $c d t-1$ (NCU00801) and lat-1 (NCU02188), were also decreased, supporting our hypothesis. It was intriguing to find that the transcription level of another key regulator, cellulose degradation regulator 1 (CLR-1), was not significantly down-regulated in the RESS condition (Additional file 4: Figure S8). CLR1 has been shown to be necessary for expression of $c l r-2$ [33] and is up-regulated when fungal cells are exposed to cellulose, suggesting the expression of $c l r-1$ may be directly regulated by biopolymer inducers while $c l r-2$ transcription is not only controlled by CLR-1 but also regulated by some other players in the ER stress response. With the discovery mentioned above, a new model for the lignocellulase induction and synthesis of N. crassa is presented (Figure 7).

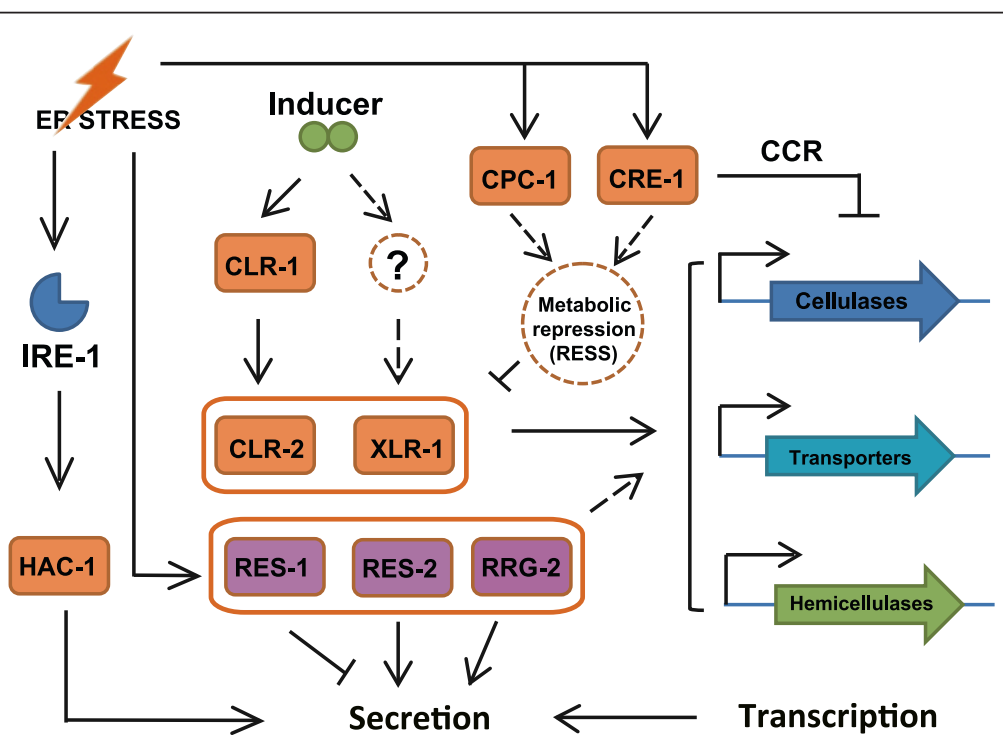

Figure 7 Schematic model illustrating the coordinated action of ER stress and cellulase synthesis. ER stress affects cellulase gene expression and secretion via the core lignocellulase regulators. Acute ER stress specifically down-regulates $\mathrm{Clr}-2$ and $x \mid r-1$ rather than $c / r-1$ for rapid adjustment of lignocellulase transcript abundance. The CRE-1-mediated CCR pathway activated by ER stress might also contribute to this process. The newly identified regulators RES-1, RES-2, and RRG-2 indirectly affect lignocellulase production. However, enzyme synthesis was increased in $\triangle$ res-1 when compared with WT, whereas $\Delta$ res-2 and $\Delta r r g-2$ showed repressive effects, suggesting RES-1 likely acts as repressor while RES-2 and RRG-2 act as activators for the synthesis and secretion of extracellular enzymes. The IRE-1/HAC-1-mediated UPR pathway also acts indirectly on lignocellulase production by mainly regulating protein folding, modification (such as glycosylation), and transport. The finding of RESS being independent of UPR pathway, raise the possibility that metabolic repression resulting from ER stress might contribute to this process. 
RESS was shown to be activated independently of the IRE-1/HAC-1 mediated UPR pathway in this work (profiling data on Additional file 1: Table S1 p2 and Figure 7). How RESS is triggered by ER stress remains to be elucidated. Notably, a previous study in T. reesei demonstrated that the intracellular concentrations of some free amino acids increased upon acute ER stress [44]. Moreover, we found cre-1 expression levels to be elevated when cells suffered ER stress, suggesting that the intracellular glucose homeostasis may also be perturbed under these conditions. These observations raise the possibility that metabolic repression that results from internal fluctuations of intracellular nutritional cues, such as simple sugars or free amino acids, might have an impact on RESS, although more data need to be acquired to support this hypothesis.

\section{Conclusions}

Here, we deeply investigated the complicated crosstalk between the ER stress response and lignocellulase synthesis in $N$. crassa by combining transcriptional profiling, mutant screening, and a detailed regulatory network analysis. The genome-wide screening uncovered hundreds of genes that might be involved in this pathway, including 100 formerly hypothetical genes. Our data furthermore allowed connecting the function of three novel transcription factors to cellulase expression and the ER stress response, shedding new light on the molecular mechanism of this complicated cross-talk in fungi. Since filamentous fungi are important systems for biotechnological enzyme production, the findings of the present study should have an impact on industrial fungal strain development for both lignocellulase production as well as synthesis of biomass-based chemicals.

\section{Materials and methods Strains}

The $N$. crassa strains used in the study were obtained from the Fungal Genetics Stock Center (http://www.fgsc. net/) [59], including the WT strain FGSC\#2489 (Mat A), FGSC\#9720 (mus-52::bar; his-3, Mat A), the cpc-1 mutant (CD15 allele mutation, FGSC\#4264) and a set of 526 homokaryotic deletion strains individually knockedout in non-essential genes encoding proteins playing potential roles in ER stress response. The hac-1 gene was deleted following standard methods provided by the Neurospora Functional Genomics Project (http://www.fgsc. net/ neurosporaprotocols/KO_Protocols.pdf, for details see Additional file 6: Supporting Information), while a homokaryotic ire-1 KO mutant was generated by a sexual cross of the heterkaryon FGSC\#21727 with wild-type strain FGSC\#2489.

\section{Liquid cultures for ER stress stimulation}

For strains able to utilize cellulose, such as WT, $\Delta$ res-1, $\Delta r e s-2$, and $\Delta r r g-2$, liquid cultivations were carried out on a defined cellulose medium $(1 \times$ Vogel's salts, $1 \% \mathrm{w} / \mathrm{v}$ crystalline cellulose (Avicel PH-101; Sigma-Aldrich, St. Louis, USA), $0.2 \% w / v \mathrm{NH}_{4} \mathrm{NO}_{3}$ ) according to published data [60]. Ten-day-old conidia were inoculated into a $50-\mathrm{mL}$ medium at $10^{6}$ conidia/mL final concentrations and grown at $25^{\circ} \mathrm{C}$ in constant light and agitation (200 rpm). After $36 \mathrm{~h}$, the cultures were treated with either dithiothreitol (DTT) or tunicamycin (TM) with indicated doses for one more hour to induce ER stress. Since both strains, $\Delta$ ire-1 and $\Delta h a c-1$, are unable to utilize cellulose, both mutants and their parental strains were pre-grown in minimal medium $(1 \times$ Vogel's salts, $2 \% w / v$ sucrose) for $16 \mathrm{~h}$, and then young hyphae were harvested and transferred into cellulose medium as mentioned above for another $4 \mathrm{~h}$ to induce cellulase gene expression. After that, $5 \mathrm{mM}$ DTT were added into each culture to stimulate ER stress for one more hour.

\section{ER stress sensitivity screening}

Fresh conidia were harvested from 10-day-old slants for each strain and suspended in sterilized water to a final concentration around $3 \times 10^{5}$ per $\mathrm{mL}$. Three microliters of a conidia suspension (approximately 1,000 conidia) were spotted onto modified solid GFS medium [61] (1× Vogel's salts, $2 \% \mathrm{w} / v$ sorbose, $0.05 \% \mathrm{w} / v$ glucose, $0.05 \%$ $w / v$ fructose, $1.5 \% w / v$ agar) including the indicated dose of DTT or TM. Colonial growth was performed at $25^{\circ} \mathrm{C}$ for 3 days. The antibiotic Hygromycin $\mathrm{B}$ was added into the medium at a final concentration of $200 \mu \mathrm{g} / \mathrm{mL}$ for all knock-out mutants, while L-histidine was added with a final concentration of $100 \mu \mathrm{g} / \mathrm{mL}$ for $\Delta h a c-1$ and FGSC\#9720.

\section{Total RNA extraction and real-time qPCR analysis}

Total RNA from frozen samples was isolated using Zirconia/Silica beads (0.5 mm diameter; Biospec Products, Bartlesville, USA) and a Mini-Beadbeater (Biospec Products, Bartlesville, USA) with $1 \mathrm{~mL}$ TRIzol reagent (Invitrogen, Grand Island, USA) according to the manufacturer's protocol. An additional clean-up including the on-column DNase I treatment was performed by using the RNeasy mini kit (QIAGEN, Valencia, USA). For qPCR analysis, reverse transcription was performed using iScript ${ }^{\mathrm{TM}} \mathrm{CDNA}$ synthesis kit (Bio-Rad, Hercules, USA). The qPCR was performed using $\mathrm{iQ}^{\text {тм }}$ SYBR Green Supermix (Bio-Rad, Hercules, USA) on the CFX connect ${ }^{\text {TM }}$ Real-Time system (Bio-Rad, Hercules, USA). PCR reaction setup was according to the manufacturer's instructions. The relative transcript level of each gene was calculated by the 2- $\Delta \Delta C t$ (comparative $\mathrm{Ct}$ ) method. Data were normalized to the endogenous control actin (NCU04173) with expression on untreated control as the reference sample. 


\section{RNA sequencing and data analysis}

The total RNA of two biological replicate samples was extracted separately, mixed together after measuring the quality of each sample, and used for high-throughput RNA sequencing. The 13 49-nt single-end RNA-seq libraries were generated commercially at Beijing Genomics Institute (BGI, Shenzhen, China) while eight 90-nt pairedend RNA-seq libraries were generated at GENEWIZ (Biotechnology Co. Ltd, Suzhou, China) by using Illumina's HiSeq $^{\text {TM }}$ 2,000 platform (Illumina, San Diego, USA) (see summary of Additional file 1: Table S1 for details). Most of the data in the present study were generated by sequencing only one sample. However, as a reference, we performed parallel sequencing in this study for WT to make sure the sequencing data we got were technically reliable. The data of libraries 01 and 14 (Additional file 1: Table S1) come from biological replicates (WT no drug control), similar as the data from libraries 03 and 15 (Additional file 1: Table S1, WT treated with $5 \mathrm{mM}$ DTT). The correlations between the two RNA-seq replicate data were analyzed and are shown in Additional file 4: Figure S9.

The sequencing data are deposited in the Gene Expression Omnibus database (GEO, http://www.ncbi.nlm.nih. gov/geo/) with accession number GSE61949. Filtered clean eads were then aligned to the latest $N$. crassa OR74A genome (version 12) [62] (http://www.broadinstitute.org/annot ation/genome/neurospora/MultiHome.html) with splicingaware aligner TopHat2 (version 2.0.12) [63]. Abundance for each transcript was calculated using the reads per kilobase per million (RPKM) [64]. Genes with altered expression was performed by using $\mathrm{R}$ package NOISeq (version 2.6.0) [65] ( $Q$ value $\geq 0.95$ or 0.90 used as threshold for single-end or paired-end libraries, respectively, which approximately corresponds to a $\mid \log _{2}$ ratio $\mid \geq 1$ ). To discover significantly expression changes between the conditions tested, only the genes with relative high transcriptional abundance (RPKM values above 20 or 15 in at least one condition for singleend or paired-end libraries, respectively [66]) were deemed as positives and went into further analysis. MIPS FunCat online tools (http://mips.helmholtz-muenchen.de/ funcatDB/) were used to functional classification of genes with altered expression. Meanwhile, manual annotation was assigned to these genes by referring to Blast hits and Pfam domain annotation (Broad version 7) to prove or verify FunCat results (for detail, see Additional file 6: Supporting Information).

\section{Growth, lignocellulase secretion, and enzyme activity assays}

Liquid cultures for cellulase production were carried out either in cellulose medium with rich nitrogen source ( $1 \times$ Vogel's salts, $2 \% w / v$ Avicel, $0.75 \% w / v$ yeast extract) or poor nitrogen source ( $1 \times$ Vogel's salts, $2 \% w / v$ Avicel). The 10-day-old conidia of tested strains were inoculated into $100 \mathrm{~mL}$ liquid media at $10^{6}$ conidia/mL final concentration and grown at $25^{\circ} \mathrm{C}$ in constant light and shaking (200 rpm) over the course of 7 days. Since the deletion strain from the TF NCU03043 had severe defects in conidiation, young hyphae were pre-grown in minimal medium for $24 \mathrm{~h}$ to accumulate biomass, which was then shifted into rich/poor nitrogen source medium and fermented for 6 days to produce lignocellulases. For switching experiments with the hyper- or hypo-secretion mutants (such as $\Delta$ res-1, $\Delta$ res-2, and $\Delta r r g-2$ ), 10-day-old conidia were inoculated into $100 \mathrm{~mL}$ minimal medium at $10^{6} \mathrm{co}-$ nidia/mL final concentration and grown at $25^{\circ} \mathrm{C}$ in constant light and agitation (200 rpm). After $16 \mathrm{~h}$, young hyphae were harvested and transferred into cellulose medium with rich nitrogen source for the indicated times to produce cellulases. Relative enzyme activity of culture supernatants was assayed with Remazol brilliant Blue R-conjugated CMC kits (Megazyme, Wicklow, Ireland). The amount of secreted protein was determined using the Bradford protein assay (Bio-Rad, Hercules, USA). For protein gel electrophoresis, 4- $\mu$ l unconcentrated culture supernatants were loaded on SDS-PAGE (Novex ${ }^{\oplus}$ NuPAGE $^{\oplus}$ Pre-cast Protein Gels, Thermo fisher scientific, Waltham, USA). Directly measuring the dry weight of fungal biomass was replaced by measuring the total protein content in order to mitigate the interference of Avicel on the test. Assays were performed as previously described [67]. To check the actual protein loading of the secretory pathway of certain mutants (for example, $\Delta$ hac-1, $\Delta$ ire-1, $\Delta$ res-1, $\Delta$ res-2, and $\Delta r r g-2)$, total protein from the same amount of biomass for each strain grown on sucrose or Avicel were extracted as previously described [67]. Unconcentrated extractions $(4 \mu \mathrm{l})$ for each sample were loaded on SDS-PAGE.

\section{Microscopy and image processing}

The growth phenotype of $\Delta$ ire-1/ $\Delta$ hac-1 as well as their parental strains was monitored by inoculating around 1,000 conidia for each strain into the center of petri dishes containing minimal medium and growth for 3 days. For recording conidial and mycelial differential interference contrast (DIC) images, cultures were performed in liquid minimal medium and samples collected at indicated time points. DIC images were recorded by using an Olympus BX51 Microscope (Olympus, Tokyo, Japan). Image Pro Plus (v6.0) was used for image processing.

\section{Statistical significance tests and data plotting}

Unless otherwise noted, all experiments were performed in triplicate and statistical tests for significance determined via one-way ANOVA by using $\mathrm{R}$ (version 3.1.1). R Packages include pheatmap (version 0.7.7) [68], ggplot2 (version 0.9.3.1) [69], VennDiagram (version 1.6.9) [70], and phenotypicForest (version 0.2) [71] used to data plotting. 


\section{Additional files} Additional file 1: Table S1. RNA-seq read profiles mapped to the
genome of $N$. crassa.

Additional file 2: Table S2. Differential expression analysis.

Additional file 3: Table S3. Functional groups of genes with altered expression in response to ER stress with different stress intensities.

Additional file 4: Figures S1-S9. Supplementary figures referred to in the main text.

Additional file 5: Table S4. Phenotypical screen of 527 ESRT KO mutants under DTT-induced ER stress.

Additional file 6: Supporting information. Additional methods referred to in the main text.

Additional file 7: Table S5. Genes regulated by either IRE-1 or HAC-1 in N. crassa.

Additional file 8: Table S6. TFs associated with ER stress response and cellulase synthesis in N. crassa.

Additional file 9: Table S7. Genes regulated by the TFS RES-1, RES-2, or RRG-2 in N. crassa.

\section{Abbreviations}

Bp: base pair; clr-1/clr-2: cellulose degradation regulator 1/2; cpc-1: cross pathway control protein 1; ER: endoplasmic reticulum; ESRTs: ER stress response targets; hac-1: homologous to ATF/CREB1; ire-1: inositol-requiring enzyme-1; Nt: nucleotide; ORF: open reading frame; res-1/res-2: regulator for ER stress response 1/2; RESS: repression under secretion stress; RPKM: reads per kilobase per million; rrg-2: response regulator 2; TF: transcription factor; UPR: unfolded protein response; xIr-1: xylan degradation regulator 1.

\section{Competing interests}

The authors declare that they have no competing interests.

\section{Authors' contributions}

FF, GM, CT, and YM designed the research; FF and GM performed the research; FF, JL, JPB, and QL analyzed the data; and FF, GM, JPB, and CT wrote the paper. All authors read and approved the final manuscript.

\section{Acknowledgements}

This work was supported by a grant from the 973 Program of China (2011CB707403 and 2011CBA00800), the 863 Project (2012AA022203D and SS2014AA021300), and the NSF of China (31171207 and 31471186). The authors wish to thank Mr. Wenliang Sun and Ms. Lixian Wang for the help in the experiments and Dr. Carolyn Rasmussen, Dr. William Beeson and Mr. Nils Thieme for critical reading of the manuscript.

\section{Author details}

${ }^{1}$ Key Laboratory of Systems Microbial Biotechnology, Tianjin Institute of Industrial Biotechnology, Chinese Academy of Sciences, Xiqi Dao32, Tianjin Airport Economic Area, Tianjin 300308, China. ${ }^{2}$ University of Chinese Academy of Sciences, Yuquan Road, Beijing 100049, China. ${ }^{3}$ Holzforschung München, TUM School of Life Sciences Weihenstephan, Technische Universität München, Hans-Carl-von-Carlowitz-Platz 2, Freising, Germany.

Received: 27 November 2014 Accepted: 27 March 2015

Published online: 14 April 2015

\section{References}

1. Himmel ME, Ding SY, Johnson DK, Adney WS, Nimlos MR, Brady JW, et al. Biomass recalcitrance: engineering plants and enzymes for biofuels production. Science. 2007;315:804-7.

2. Yan S, Wu G. Secretory pathway of cellulase: a mini-review. Biotechnol Biofuels. 2013;6:177-88.

3. Ghosh A, Al-Rabiai S, Ghosh BK, Trimiño-Vazquez H, Eveleigh DE, Montenecourt BS. Increased endoplasmic reticulum content of a mutant of Trichoderma reesei (Rut-C30) in relation to cellulase synthesis. Enzyme Microb Technol. 1982;4:110-3.
4. Ghosh A, Ghosh BK, Trimiño-Vazquez H, Eveleigh DE, Montenecourt BS. Cellulase secretion from a hypercellulolytic mutant of Trichoderma reesei Rut-C30. Arch Microbiol. 1984;140:126-33.

5. Pakula TM, Salonen K, Uusitalo J, Penttila M. The effect of specific growth rate on protein synthesis and secretion in the filamentous fungus Trichoderma reesei. Microbiology. 2005;151:135-43.

6. Ron D, Walter P. Signal integration in the endoplasmic reticulum unfolded protein response. Nat Rev Mol Cell Biol. 2007;8:519-29.

7. Hetz C. The unfolded protein response: controlling cell fate decisions under ER stress and beyond. Nat Rev Mol Cell Biol. 2012;13:89-102.

8. Hollien J. Evolution of the unfolded protein response. Biochim Biophys Acta. 2013;1833:2458-63.

9. Pakula TM, Laxell M, Huuskonen A, Uusitalo J, Saloheimo M, Penttila M. The effects of drugs inhibiting protein secretion in the filamentous fungus Trichoderma reesei. Evidence for down-regulation of genes that encode secreted proteins in the stressed cells. J Biol Chem. 2003:278:45011-20.

10. Al-Sheikh H, Watson AJ, Lacey GA, Punt PJ, MacKenzie DA, Jeenes DJ, et al. Endoplasmic reticulum stress leads to the selective transcriptional downregulation of the glucoamylase gene in Aspergillus niger. Mol Microbiol. 2004:53:1731-42.

11. Carvalho ND, Jørgensen TR, Arentshorst M, Nitsche BM, van den Hondel CA, Archer DB, et al. Genome-wide expression analysis upon constitutive activation of the HacA bZIP transcription factor in Aspergillus niger reveals a coordinated cellular response to counteract ER stress. BMC Genomics. 2012;13:350-66.

12. Glass NL, Schmoll M, Cate JH, Coradetti S. Plant cell wall deconstruction by ascomycete fungi. Annu Rev Microbiol. 2013;67:477-98.

13. Znameroski EA, Glass NL. Using a model filamentous fungus to unravel mechanisms of lignocellulose deconstruction. Biotechnol Biofuels. 2013;6:6-12

14. Tian C, Beeson WT, lavarone AT, Sun J, Marletta MA, Cate JH, et al. Systems analysis of plant cell wall degradation by the model filamentous fungus Neurospora crassa. Proc Natl Acad Sci U S A. 2009;106:22157-62.

15. Benz JP, Chau BH, Zheng D, Bauer S, Glass NL, Somerville CR. A comparative systems analysis of polysaccharide-elicited responses in Neurospora crassa reveals carbon source-specific cellular adaptations. Mol Microbiol. 2014;91:275-99.

16. Colot HV, Park G, Turner GE, Ringelberg C, Crew CM, Litvinkova L, et al. A high-throughput gene knockout procedure for Neurospora reveals functions for multiple transcription factors. Proc Natl Acad Sci U S A. 2006;103:10352-7.

17. Xu C, Bailly-Maitre B, Reed JC. Endoplasmic reticulum stress: cell life and death decisions. J Clin Invest. 2005;115:2656-64.

18. Travers KJ, Patil CK, Wodicka L, Lockhart DJ, Weissman JS, Walter P. Functional and genomic analyses reveal an essential coordination between the unfolded protein response and ER-associated degradation. Cell. 2000;101:249-58.

19. Ruepp A, Zollner A, Maier D, Albermann K, Hani J, Mokrejs M, et al. The FunCat, a functional annotation scheme for systematic classification of proteins from whole genomes. Nucleic Acids Res. 2004;32:5539-45.

20. Paluh JL, Plamann M, Kruger D, Barthelmess IB, Yanofsky C, Perkins DD. Determination of the inactivating alterations in two mutant alleles of the Neurospora crassa cross-pathway control gene cpc-1. Genetics. 1990;124:599-606

21. Barnes G, Hansen WJ, Holcomb CL, Rine J. Asparagine-linked glycosylation in Saccharomyces cerevisiae: genetic analysis of an early step. Mol Cell Biol. 1984;4:2381-8

22. Lin JH, Li H, Yasumura D, Cohen HR, Zhang C, Panning B, et al. IRE1 signaling affects cell fate during the unfolded protein response. Science. 2007;318:944-9.

23. Nagashima Y, Mishiba K, Suzuki E, Shimada Y, Iwata Y, Koizumi N. Arabidopsis IRE1 catalyses unconventional splicing of bZIP60 mRNA to produce the active transcription factor. Sci Rep. 2011;1:29.

24. Hooks KB, Griffiths-Jones S. Conserved RNA structures in the non-canonical Hac1/Xbp1 intron. RNA Biol. 2011;8:552-6.

25. Feng X, Krishnan K, Richie DL, Aimanianda V, Hartl L, Grahl N, et al. HacA-independent functions of the ER stress sensor IreA synergize with the canonical UPR to influence virulence traits in Aspergillus fumigatus. PLoS Pathog. 2011;7, e1002330. 
26. Kimata Y, Ishiwata-Kimata Y, Yamada S, Kohno K. Yeast unfolded protein response pathway regulates expression of genes for anti-oxidative stress and for cell surface proteins. Genes Cells. 2006;11:59-69.

27. Shen X, Ellis RE, Sakaki K, Kaufman RJ. Genetic interactions due to constitutive and inducible gene regulation mediated by the unfolded protein response in C. elegans. PLoS Genet. 2005;1, e37.

28. Wimalasena T, Enjalbert B, Guillemette T, Plumridge A, Budge S, Yin Z, et al. Impact of the unfolded protein response upon genome-wide expression patterns, and the role of Hac1 in the polarized growth, of Candida albicans. Fungal Genet Biol. 2008;45:1235-47.

29. Wakasa Y, Oono Y, Yazawa T, Hayashi S, Ozawa K, Handa H, et al. RNA sequencing-mediated transcriptome analysis of rice plants in endoplasmic reticulum stress conditions. BMC Plant Biol. 2014;14:101.

30. Shelest E. Transcription factors in fungi. FEMS Microbiol Lett. 2008;286:145-51.

31. Sun J, Glass NL. Identification of the CRE-1 cellulolytic regulon in Neurospora crassa. PLoS One. 2011;6, e25654.

32. Banno S, Noguchi R, Yamashita K, Fukumori F, Kimura M, Yamaguchi I, et al. Roles of putative His-to-Asp signaling modules HPT-1 and RRG-2, on viability and sensitivity to osmotic and oxidative stresses in Neurospora crassa. Curr Genet. 2007:51:197-208.

33. Coradetti ST, Craig JP, Xiong Y, Shock T, Tian C, Glass NL. Conserved and essential transcription factors for cellulase gene expression in ascomycete fungi. Proc Natl Acad Sci U S A. 2012;109:7397-402.

34. Sun J, Tian C, Diamond S, Glass NL. Deciphering transcriptional regulatory mechanisms associated with hemicellulose degradation in Neurospora crassa. Eukaryot Cell. 2012;11:482-93.

35. Znameroski EA, Coradetti ST, Roche CM, Tsai JC, lavarone AT, Cate JH, et al. Induction of lignocellulose-degrading enzymes in Neurospora crassa by cellodextrins. Proc Natl Acad Sci U S A. 2012;109:6012-7.

36. Pehar M, Jonas MC, Hare TM, Puglielli L. SLC33A1/AT-1 protein regulates the induction of autophagy downstream of IRE1/XBP1 pathway. J Biol Chem. 2012;287:29921-30.

37. Jonikas MC, Collins SR, Denic V, Oh E, Quan EM, Schmid V, et al. Comprehensive characterization of genes required for protein folding in the endoplasmic reticulum. Science. 2009;323:1693-7.

38. Dombroski BA, Nayak RR, Ewens KG, Ankener W, Cheung VG, Spielman RS. Gene expression and genetic variation in response to endoplasmic reticulum stress in human cells. Am J Hum Genet. 2010;86:719-29.

39. Chow CY, Wolfner MF, Clark AG. Using natural variation in Drosophila to discover previously unknown endoplasmic reticulum stress genes. Proc Natl Acad Sci U S A. 2013;110:9013-8.

40. Mulder HJ, Nikolaev I. HacA-dependent transcriptional switch releases hacA mRNA from a translational block upon endoplasmic reticulum stress. Eukaryot Cell. 2009;8:665-75.

41. Ruegsegger $\mathrm{U}$, Leber $\mathrm{JH}$, Walter P. Block of HAC1 mRNA translation by longrange base pairing is released by cytoplasmic splicing upon induction of the unfolded protein response. Cell. 2001;107:103-14.

42. Yoshida H, Oku M, Suzuki M, Mori K. pXBP1(U) encoded in XBP1 pre-mRNA negatively regulates unfolded protein response activator $\mathrm{PXBP1(S)}$ in mammalian ER stress response. J Cell Biol. 2006;172:565-75.

43. Tian C, Kasuga T, Sachs MS, Glass NL. Transcriptional profiling of cross pathway control in Neurospora crassa and comparative analysis of the Gcn4 and CPC1 regulons. Eukaryot Cell. 2007;6:1018-29.

44. Arvas M, Pakula $T$, Lanthaler $K$, Saloheimo M, Valkonen M, Suortti $T$, et al. Common features and interesting differences in transcriptional responses to secretion stress in the fungi Trichoderma reesei and Saccharomyces cerevisiae. BMC Genomics. 2006;7:32.

45. Wang B, Guo G, Wang C, Lin Y, Wang $X$, Zhao M, et al. Survey of the transcriptome of Aspergillus oryzae via massively parallel mRNA sequencing. Nucleic Acids Res. 2010;38:5075-87.

46. Soriani FM, Malavazi I, da Silva Ferreira ME, Savoldi M, Von Zeska Kress MR, de Souza Goldman MH, et al. Functional characterization of the Aspergillus fumigatus CRZ1 homologue CrzA. Mol Microbiol. 2008;67:1274-91.

47. Kwon NJ, Garzia A, Espeso EA, Ugalde U, Yu JH. FlbC is a putative nuclear $\mathrm{C} 2 \mathrm{H} 2$ transcription factor regulating development in Aspergillus nidulans. Mol Microbiol. 2010;77:1203-19.

48. Wendland J, Walther A. Tip growth and endocytosis in fungi. In: Samaj J, Baluska F, Menzel D, editors. The plant endocytosis 2005. Heidelberg, Germany: Springer; 2005. p. 293-310.
49. Silverman-Gavrila LB, Lew RR. Calcium gradient dependence of Neurospora crassa hyphal growth. Microbiology. 2003;149:2475-85.

50. Bowman BJ, Abreu S, Margolles-Clark E, Draskovic M, Bowman EJ. Role of four calcium transport proteins, encoded by nca-1, nca-2, nca-3, and cax, in maintaining intracellular calcium levels in Neurospora crassa. Eukaryot Cell. 2011;10:654-61.

51. Lee J, Godon C, Lagniel G, Spector D, Garin J, Labarre J, et al. Yap1 and Skn7 control two specialized oxidative stress response regulons in yeast. J Biol Chem. 1999;274:16040-6.

52. Delic M, Rebnegger C, Graf AB, Mattanovich D, Gasser B. Overexpression of the redox transcription factor Yap1 modifies intracellular redox conditions and enhances recombinant protein secretion. Microbial Cell. 2013;11:376-86.

53. Malhotra JD, Miao H, Zhang K, Wolfson A, Pennathur S, Pipe SW, et al. Antioxidants reduce endoplasmic reticulum stress and improve protein secretion. Proc Natl Acad Sci U S A. 2008;105:18525-30.

54. Park G, Ryu YH, Hong YJ, Choi EH, Uhm HS. Cellular and molecular responses of Neurospora crassa to non-thermal plasma at atmospheric pressure. Appl Phys Lett. 2012;100:063703.

55. Lewis TL, Keesler GA, Fenner GP, Parks LW. Pleiotropic mutations in Saccharomyces cerevisiae affecting sterol uptake and metabolism. Yeast. 1988:4:93-106.

56. Pineau L, Colas J, Dupont S, Beney L, Fleurat-Lessard P, Berjeaud JM, et al. Lipid-induced ER stress: synergistic effects of sterols and saturated fatty acids. Traffic. 2009;10:673-90.

57. Aro N, Ilmen M, Saloheimo A, Penttila M. ACEl of Trichoderma reesei is a repressor of cellulase and xylanase expression. Appl Environ Microbiol. 2003;69:56-65

58. Chilton IJ, Delaney CE, Barham-Morris J, Fincham DA, Hooley P, Whitehead MP. The Aspergillus nidulans stress response transcription factor StzA is ascomycete-specific and shows species-specific polymorphisms in the C-terminal region. Mycol Res. 2008;112:1435-46.

59. McCluskey K. The fungal genetics stock center: from molds to molecules. Adv Appl Microbiol. 2003;52:245-62.

60. Yazdi MT, Woodward JR, Radford A. Cellulase production by Neurospora-crassa the enzymes of the complex and their regulation. Enzyme Microb Technol. 1990;12:116-9.

61. Davis RH, de Serres FJ. Genetic and microbiological research techniques for Neurospora crassa. Methods Enzymol. 1970;17A:79-143.

62. Galagan JE, Calvo SE, Borkovich KA, Selker EU, Read ND, Jaffe D, et al. The genome sequence of the filamentous fungus Neurospora crassa. Nature. 2003:422:859-68.

63. Kim D, Pertea G, Trapnell C, Pimentel H, Kelley R, Salzberg SL. TopHat2: accurate alignment of transcriptomes in the presence of insertions, deletions and gene fusions. Genome Biol. 2013;14:R36.

64. Mortazavi A, Williams BA, Mccue K, Schaeffer L, Wold B. Mapping and quantifying mammalian transcriptomes by RNA-Seq. Nat Methods. 2008:5:621-8.

65. Tarazona S, Garcia-Alcalde F, Dopazo J, Ferrer A, Conesa A. Differential expression in RNA-seq: A matter of depth. Genome Res. 2011;21:2213-23.

66. McIntyre LM, Lopiano KK, Morse AM, Amin V, Oberg AL, Young L, et al. RNA-seq : technical variability and sampling. BMC Genomics. 2011;12:293.

67. Brown NA, de Gouvea PF, Krohn NG, Savoldi M, Goldman GH. Functional characterisation of the non-essential protein kinases and phosphatases regulating Aspergillus nidulans hydrolytic enzyme production. Biotechnol Biofuels. 2013;6:91

68. Kolde R. pheatmap: Pretty Heatmaps. 2011. R package version 0.5.1. [http://CRAN.R-project.org/package=pheatmap].

69. Ginestet C. ggplot2: elegant graphics for data analysis. J R Stat Soc Ser A-Stat Soc. 2011;174:245

70. Chen H, Boutros PC. VennDiagram: a package for the generation of highly-customizable Venn and Euler diagrams in R. Bmc Bioinformatics. 2011;12:35.

71. Ladroue C. phenotypicForest. 2012. R package version 0.2 [http://chrisladroue.com/phorest/]. 